
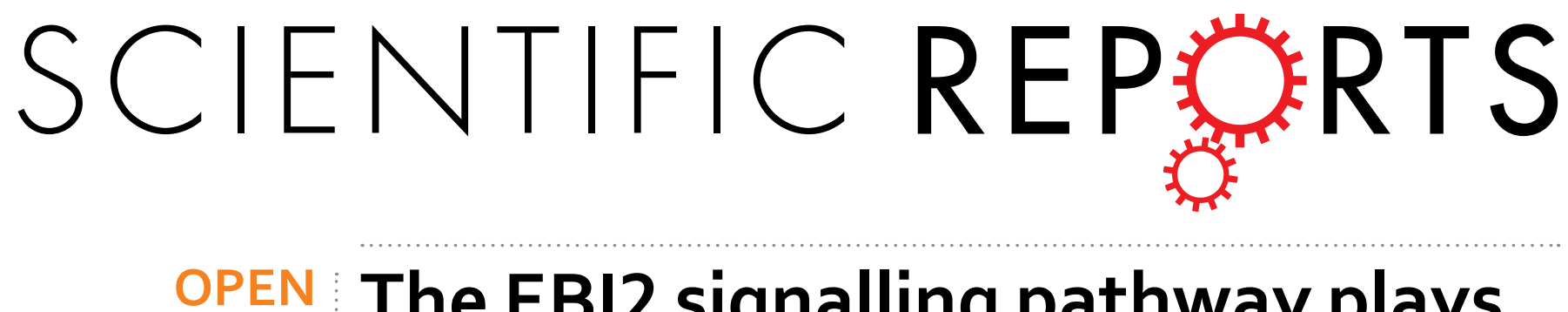

\title{
The EBI2 signalling pathway plays a role in cellular crosstalk between astrocytes and macrophages
}

Received: 10 July 2015

Accepted: 18 April 2016

Published: 11 May 2016

\author{
Aleksandra Rutkowska ${ }^{1}$, Sinead A. O’Sullivan ${ }^{1}$, Isabelle Christen², Juan Zhang ${ }^{2}$, \\ Andreas W. Sailer ${ }^{3}$ \& Kumlesh K. Dev ${ }^{1}$
}

$\mathrm{EBI} 2$ is a $\mathrm{G}$ protein-coupled receptor activated by oxysterol $7 \alpha, 25$-dihydroxycholesterol $(7 \alpha 25 \mathrm{HC})$ and regulates $\mathrm{T}$ cell-dependant antibody response and $\mathrm{B}$ cell migration. We recently found $\mathrm{EBI} 2$ is expressed in human astrocytes, regulates intracellular signalling and modulates astrocyte migration. Here, we report that LPS treatment of mouse astrocytes alters mRNA levels of EBI2 and oxysterols suggesting that the EBI2 signalling pathway is sensitive to LPS-mediated immune challenge. We also find that conditioned media obtained from LPS-stimulated mouse astrocytes induces macrophage migration, which is inhibited by the EBI2 antagonist NIBR189. These results demonstrate a role for the EBI2 signalling pathway in astrocytes as a sensor for immune challenge and for communication with innate immune cells such as macrophages.

EBI2 (Epstein-Barr virus-induced gene 2, GPR183) is a G protein-coupled receptor that is expressed in peripheral blood mononuclear cells ${ }^{1-3}$ and activated by the oxysterol $7 \alpha 25 \mathrm{HC}(7 \alpha, 25 \text {-dihydroxycholesterol })^{4}$. EBI2 regulates $\mathrm{B}$ cell positioning in lymphoid tissue and is crucial for launching T cell-dependant antibody response ${ }^{5,6}$. EBI2 is coupled exclusively to the $G_{i}$ protein and when activated signals via two mitogen-activated protein (MAP) kinases, p38 and extracellular-signal-regulated kinase (ERK), as well as calcium and serum response element (SRE) transcription factor in a pertussis toxin (Ptx)-sensitive manner ${ }^{1,3,7-9}$. Notably, it does not signal via nuclear factor of activated T cells (NFAT) or nuclear factor kappa B $(\mathrm{NF} \kappa \mathrm{B})^{7,8}$. The most potent EBI2 agonist, $7 \propto 25 \mathrm{HC}$, is synthesised from cholesterol by the enzymes cholesterol 25 -hydroxylase $(\mathrm{CH} 25 \mathrm{H})$ and cytochrome P450 oxysterol 7-alpha-hydroxylase (CYP7B1) and is degraded by cholest-5-ene-3 $\beta, 7 \alpha$-diol $3 \beta$-dehydrogenase (HSD3B7) ${ }^{4,10}$. Upon challenge with lipopolysaccharide (LPS), B cells, macrophages, and other immune cells upregulate expression of EBI2 and the CYP7B1 and CH25H enzymes, while decreasing HSD3B7, thus apparently promoting EBI2 signalling ${ }^{11,12}$. It is noteworthy that such LPS immune challenge can also increase the levels of $25 \mathrm{HC}$ (the precursor to $7 \alpha 25 \mathrm{HC}$ ) in the humans and mice ${ }^{11,13}$ and moreover, that $25 \mathrm{HC}$ inhibits activity of human immunodeficiency virus, herpes simplex virus 1 and Ebola virus ${ }^{14}$. Thus, the induction of oxysterols in response to TLR4 stimulation or type I/II interferons might be a host strategy to fight such infections ${ }^{11,15}$, where EBI2 signalling may play a role.

The potential roles of the EBI2 signalling pathway molecules in disease and their potential uses as drug targets for Epstein-Barr virus (EBV) infection and EBV-mediated diseases as well as type-1-diabetes, multiple sclerosis, rheumatoid arthritis and systemic lupus erythematosus has been recently discussed ${ }^{16-20}$. For example, it has been shown that animals deficient in the $7 \alpha 25 \mathrm{HC}$ synthesising enzyme, $\mathrm{CH} 25 \mathrm{H}$, exhibit worsened course of experimental allergic encephalomyelitis (EAE) and that $\mathrm{CH} 25 \mathrm{H}$ knock-out $(\mathrm{KO})$ macrophages have a proinflammatory phenotype compared to wild-type (WT $)^{21}$. In agreement with the idea that EBI2 signalling may play a direct role in the central nervous system (CNS), we have reported that EBI2 is expressed in astrocytes, regulates astrocyte signalling, as well as astrocyte cell migration ${ }^{22}$. Another link between EBI2 signalling and brain disease comes from findings that CYP7B1/SPG5 mutations result in type 5 hereditary spastic paraplegia (hSPG5) ${ }^{23}$. Notably, in this illness, deficits in axonal trafficking, altered myelination state and neurodegeneration have been observed, suggesting a dysfunction in EBI2 signalling may induce brain pathophysiology as well as aberrant immune cell

${ }^{1}$ Drug Development, School of Medicine, Trinity College, Dublin, Ireland. ${ }^{2}$ Analytical Sciences and Imaging, Novartis Institutes for BioMedical Research, Novartis Pharma AG, Basel, Switzerland. ${ }^{3}$ Developmental and Molecular Pathways, Novartis Institutes for BioMedical Research, Novartis Pharma AG, Basel, Switzerland. Correspondence and requests for materials should be addressed to K.K.D. (email: devk@tcd.ie) 
a

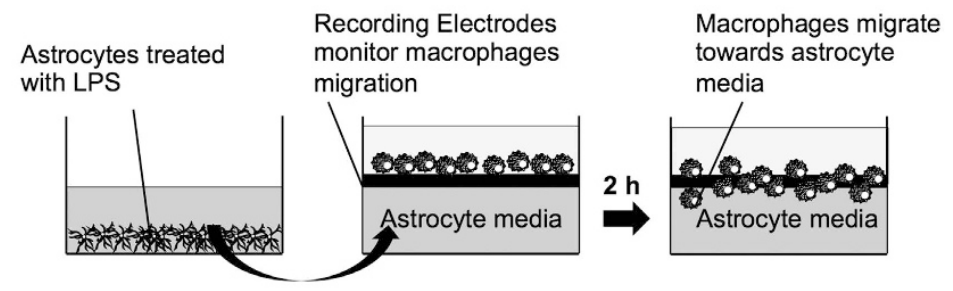

b

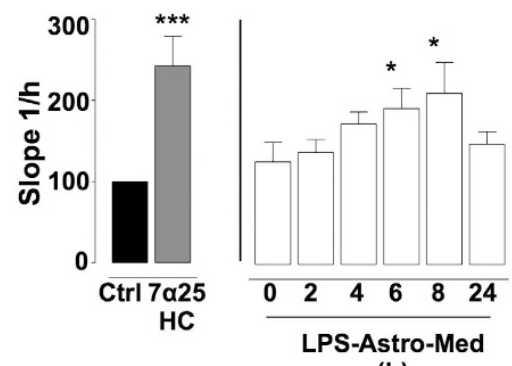

(h)

Figure 1. Conditioned media from LPS-treated mouse astrocytes induces migration of macrophage cells (RAW264.7). (a) Schematic of experimental setup. Transwell system where macrophages (RAW264.7) were plated on recording electrodes (upper chamber) and mouse astrocyte conditioned media with or without $7 \alpha 25 \mathrm{HC}$ were added to the lower chamber. The migration of macrophages from the upper chamber to the lower chamber was measured by the recording electrodes two hours after addition of astrocyte media or drugs to the bottom chamber. (b) Treatment with $7 \alpha 25 \mathrm{HC}(0.01 \mu \mathrm{M}, 2$ hours) induced a significant increase in macrophage migration. Astrocyte media collected after 6 and 8 hours of LPS $(100 \mathrm{ng} / \mathrm{ml})$ treatment induced macrophage (RAW264.7) migration while media from non-treated astrocytes or treated with LPS for 0,2 , 4, and 24 hours did not induce macrophage migration. Data presented as mean $+/-$ SEM, $n=3-5$, one-way ANOVAs with Dunnett's post hoc tests, ${ }^{\star} \mathrm{p}<0.05,{ }^{* *} \mathrm{p}<0.001$ vs. corresponding control.

function. Whether mutations in CYP7B1 result in reduced levels of $7 \alpha 25 \mathrm{HC}$ and subsequent reduced EBI2 activation in patients with hSPG5 and whether astrocytes play a specific role in this disease remains, however, to be clarified.

Here, to investigate further roles of the EBI2 signalling pathway in astrocytes, we examined its modulation by LPS in mouse astrocytes. We also examined if EBI2 signalling plays a role in the cellular communication of astrocytes with macrophages.

\section{Results}

LPS-stimulated mouse astrocyte conditioned media induces migration of macrophages. There is a growing body of evidence demonstrating that astrocytes produce a number of signalling molecules (cytokines, chemokines, growth factors, nitric oxide and others) that can allow for communication with innate immune cells and perhaps promote their migration ${ }^{24}$. We first aimed to demonstrate that astrocytes stimulated with LPS could induce the migration of innate immune cells and, in this study, focused on their ability to regulate the migration of macrophages. Media supplemented with $7 \alpha 25 \mathrm{HC}(0.01 \mu \mathrm{M})$ or taken from LPS $(100 \mathrm{ng} / \mathrm{ml}, 0-24 \mathrm{~h})$ treated mouse astrocytes (LPS-Astro-Med) was applied to the lower chamber of the xCELLigence ${ }^{\circledR}$ migration transwell system containing macrophage RAW264.7 cells in the upper chamber (Fig. 1a). This LPS-Astro-Med induced significant migration of macrophage RAW264.7 cells in a time-dependent manner, compared to non-treated astrocytes $(192.2 \%+/-50.2 \%$ at 6 hours, $211.0 \%+/-66.5 \%$ at 8 hours) (Fig. 1b). Notably, media from astrocytes treated with LPS for 2, 4 and 24 hours did not induce macrophage migration, indicating a temporal response and also indicating that the added LPS itself did not directly alter macrophage migration in these set of experiments (Fig. 1b).

LPS regulates mRNA levels of the EBI2 signalling pathway in mouse astrocytes. Secondly, we aimed to determine whether mouse astrocytes respond to inflammatory insults (LPS) by regulating the EBI2 signalling pathway (receptor and signalling molecules). We and others have previously demonstrated that LPS alters mRNA levels of EBI2 and the 7a25HC synthesising and degrading enzymes (Fig. 2a $)^{11,12,25}$. In accordance, here we found LPS suppressed EBI2 mRNA expression in mouse astrocytes (Fig. 2b). In addition, LPS induced expression of both $7 \alpha 25 \mathrm{HC}$ synthesising enzymes namely $\mathrm{CH} 25 \mathrm{H}$ and $\mathrm{CYP} 7 \mathrm{~B} 1$. The $\mathrm{CH} 25 \mathrm{H}$ mRNA peaked after $4-6$ hours $(631.5 \%+/-224.8 \%$ at 4 hours and $606.3 \%+/-335.1 \%$ at 6 hours) (Fig. 2 c). We also found LPS to increase CYP7B1 mRNA expression, with notable increases observed at 6 hours $(187.7 \%+/-120.9 \%)$ and 8 hours $(421.5 \%+/-312.9 \%)$, with significant increase at 24 hours $(635.8 \%+/-436.8 \%)$ (Fig. $2 \mathrm{~d})$. The mRNA levels of the $7 \alpha 25 \mathrm{HC}$ degrading enzyme HSD3B7 remained constant for the first 8 hours of LPS treatment and 
a
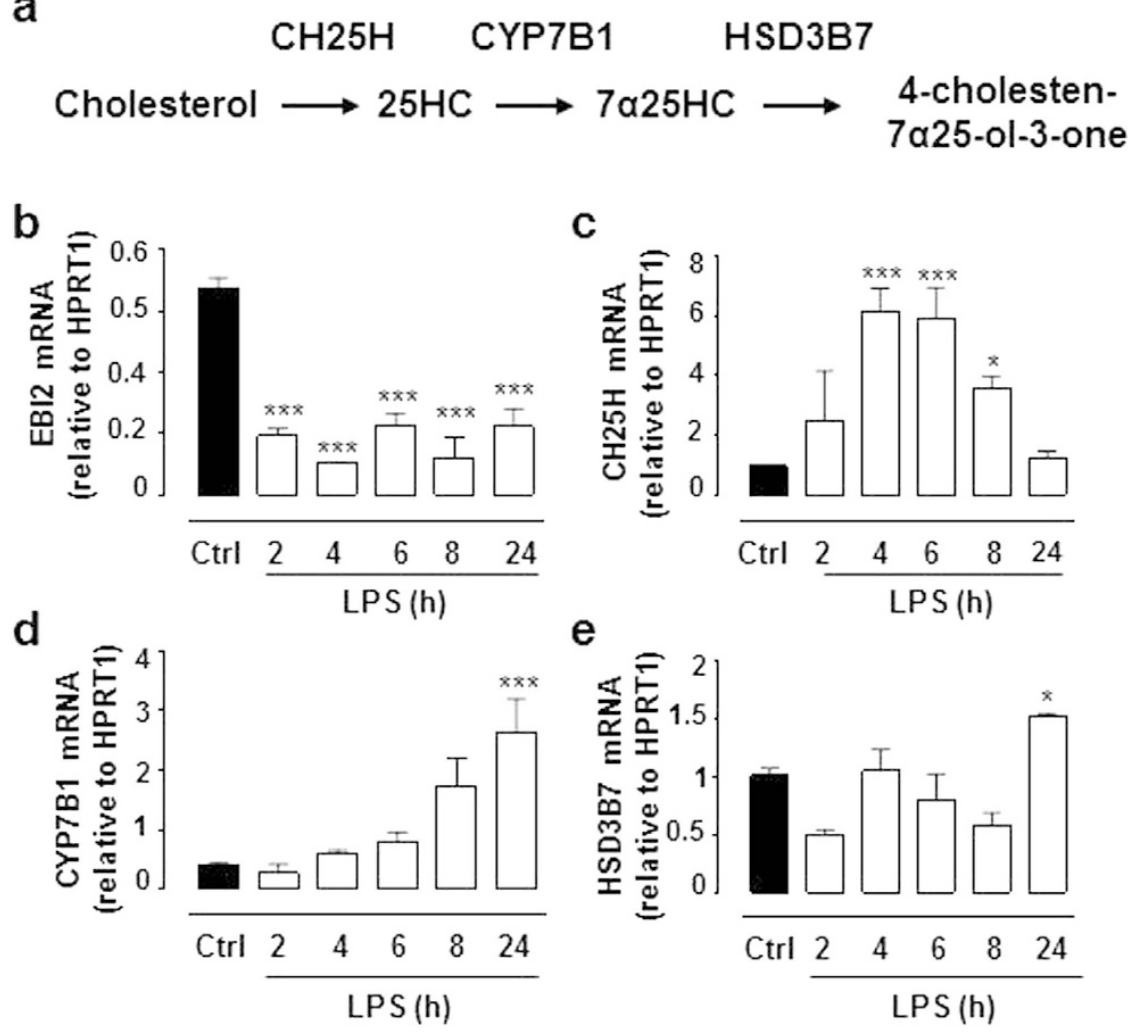

Figure 2. LPS modulates mRNA expression of molecules in the EBI2 signaling pathway in mouse astrocytes. (a) Synthetic and degrading pathway of $7 \alpha 25 \mathrm{HC}$. (b) Treatment of mouse astrocytes with LPS $(100 \mathrm{ng} / \mathrm{ml})$ induces a rapid and lasting suppression of EBI2 mRNA expression. (c) LPS induces CH25H mRNA expression in mouse astrocytes that peaks after four to six hours and declines thereafter. (d) LPS induces a slow and gradual increase in CYP7B1 mRNA expression in mouse astrocytes. (e) LPS induces increase in HSD3B7 mRNA expression after 24 hour treatment. Data presented as mean $+/-\mathrm{SEM}, \mathrm{n}=3$, one-way ANOVA and Dunnett's post-test, ${ }^{*} \mathrm{p}<0.05,{ }^{* *} \mathrm{p}<0.001$ vs. corresponding control.

increased after 24 hours ( $150.3 \%+/-6.6 \%$ at 24 hours) (Fig. 2e). These results demonstrate that molecules of the EBI2 signalling pathway in astrocytes are sensitive to immune challenge such as LPS and support the idea that EBI2 may be involved in sensing infection in both immune and central nervous systems.

LPS induces oxysterol release in mouse astrocytes. Thirdly, we examined if the LPS-induced changes in the $7 \propto 25 \mathrm{HC}$ synthesising and degrading enzymes were accompanied by altered levels of oxysterols in the astrocyte conditioned media. The levels of monohydroxylated oxysterols such as $25 \mathrm{HC}$ are induced in the CNS in human and animal subjects after treatment with LPS ${ }^{11,13}$. The release of these oxysterols has been attributed to immune cells, such as macrophages ${ }^{11-13}$. To demonstrate that astrocytes also produce oxysterols, mouse astrocytes were treated with LPS for 0-24hours and levels of monohydroxylated (24SHC, 25HC and 27HC) and dihydroxylated $(7 \alpha 24 \mathrm{SHC}, 7 \alpha 25 \mathrm{HC}, 7 \beta 25 \mathrm{HC}, 7 \alpha 27 \mathrm{HC}$ and $7 \beta 27 \mathrm{HC})$ oxysterols were measured with mass spectroscopy. The data showed no increase after LPS treatment in the levels of the following mono- and dihydroxylated oxysterols in the cell pellet: $24 \mathrm{SHC}, 7 \alpha 24 \mathrm{SHC}, 27 \mathrm{HC}, 7 \alpha 27 \mathrm{HC}, 7 \beta 27 \mathrm{HC}$ (data not shown). There was also no significant increase in the levels of mono- and dihydroxylated oxysterols $(24 \mathrm{SHC}, 7 \alpha 24 \mathrm{SHC}, 25 \mathrm{HC}, 7 \alpha 25 \mathrm{HC}, 7 \beta 25 \mathrm{HC}$, $27 \mathrm{HC}, 7 \propto 27 \mathrm{HC}$ and $7327 \mathrm{HC}$ ) in cell culture media after $0-24 \mathrm{~h}$ (data not shown). In contrast, LPS induced a significant increase in the levels of $25 \mathrm{HC}$ (the precursor to the EBI2 agonist $7 \alpha 25 \mathrm{HC})$ after four $(2,435 \%+/-$ $1,224 \%)$ and eight $(2,087 \%+/-517 \%)$ hours of treatment in mouse astrocytes as detected in the cell pellets (Fig. 3a). The levels of the EBI2 agonist $7 \alpha 25 \mathrm{HC}$ were also significantly induced in cell pellets after eight ( $4 \%$ $+/-1 \%)$ and $15(24 \%+/-5 \%)$ hours of LPS treatment (Fig. 3b). Likewise, levels of the closely related oxysterol $7325 \mathrm{HC}$ were induced after LPS stimulation in the cell pellets after four $(316 \%+/-158 \%)$, eight $(370 \%+/-$ $85 \%)$ and $15(815 \%+/-318 \%)$ hours (Fig. 3c). Together, this data, for the first time, demonstrates that astrocytes respond to the immune challenge by regulating EBI2 and oxysterol levels.

EBI2 regulates macrophage migration induced by mouse astrocyte conditioned media. Lastly, to investigate whether the observed migratory effects induced by LPS-Astro-Med were EBI2 dependant, the media was supplemented with the EBI2 antagonist (NIBR189 ref. [30], 10 $\mu \mathrm{M}$ ). The effects of oxysterols on macrophage migration have been shown to be partially inhibited with EBI2 antagonist (NIBR189) indicating EBI2 
a

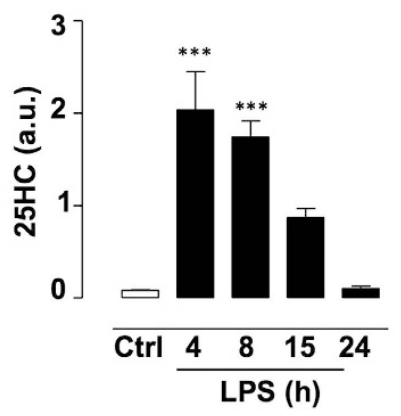

b

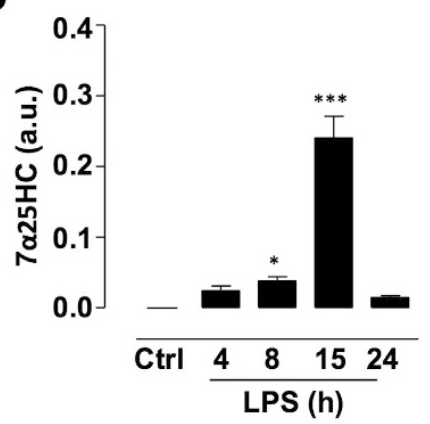

C

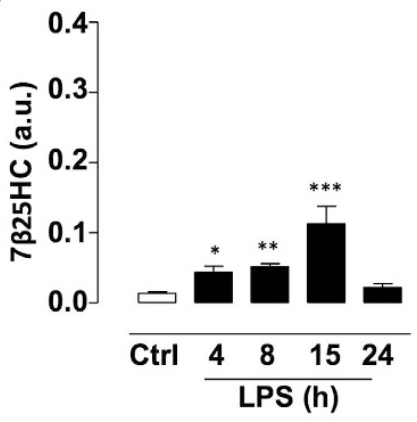

Figure 3. LPS induces oxysterol release in mouse astrocytes. Treatment of primary mouse astrocytes with LPS (100 ng/ml) induced levels of 25HC after four and eight hours (a) $7 \alpha 25 \mathrm{HC}$ after eight and 15 hours (b) and $7325 \mathrm{HC}$ after four, eight and 15 hours $(\mathbf{c})$. Data presented as mean $+/-$ SEM, $\mathrm{n}=3-6$. one-way ANOVAs with Dunnett's post-tests, ${ }^{\star} \mathrm{p}>0.05,{ }^{* *} \mathrm{p}>0.01,{ }^{* * *} \mathrm{p}>0.001$ vs corresponding control.

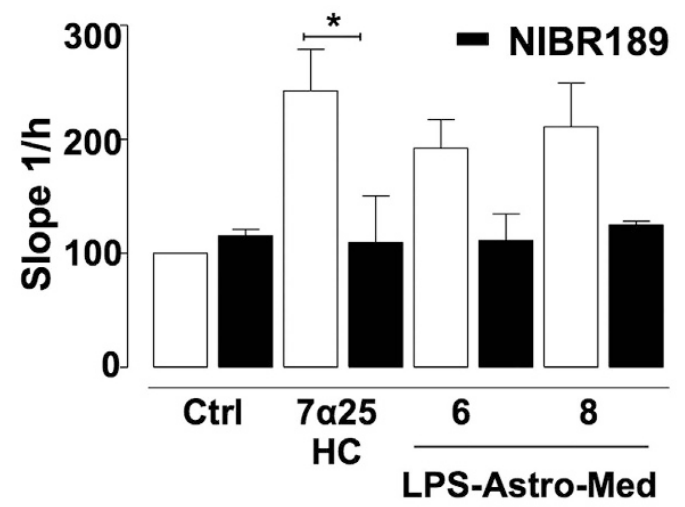

(h)

Figure 4. Macrophage (RAW264.7) migration induced by conditioned media from LPS-treated mouse astrocytes is reduced by EBI2 antagonism. Please see Fig. 1a for experimental setup. Both $7 \alpha 25 \mathrm{HC}$ $(0.01 \mu \mathrm{M})$ and astrocyte conditioned media obtained from 6 and 8 hour LPS-treated mouse astrocytes induced macrophage migration. Addition of the EBI2 antagonist (NIBR189, $10 \mu \mathrm{M}$ ) to $7 \alpha 25 \mathrm{HC}$ or the LPStreated mouse astrocyte media attenuated astrocyte conditioned media effects on macrophage migration. Data presented as mean $+/-$ SEM $\mathrm{n}=3$, one-way ANOVAs with Bonferroni's post-tests, ${ }^{*} \mathrm{p}<0.05$, vs. corresponding control.

mediated signalling ${ }^{12}$. In agreement with this previous study ${ }^{12}$, the EBI2 antagonist NIBR189 inhibited the effects of $7 \alpha 25 \mathrm{HC}$ on macrophage migration $(109.5 \%+/-57.9 \%)$, that was exogenously applied to the lower chamber of the xCELLigence ${ }^{\circledR}$ migration transwell system. Importantly, conditioned media taken from astrocytes stimulated with LPS for 6 and 8 hours (LPS-Astro-Med) and supplemented with EBI2 antagonist (NIBR189) attenuated the observed macrophage migration (Fig. 4). A trend decrease for this effect was observed suggesting partial EBI2 involvement in regulation of macrophage migration (Fig. 4). Overall, therefore, the data suggests that astrocytes stimulated with LPS can induce macrophage migration and, moreover, that EBI2 can regulate this cellular crosstalk.

\section{Discussion}

Previous research has shown conflicting results on the role of $\mathrm{CH} 25 \mathrm{H}$ in neuroinflammation. While Reboldi et al. ${ }^{21}$ showed that $\mathrm{Ch} 25 \mathrm{~h}$ deficient mouse macrophages adopt a pro-inflammatory phenotype and animals show a aggravated course of EAE, Chalmin and colleagues ${ }^{26}$ found that Ch25h deletion significantly attenuated EAE disease course by limiting trafficking of pathogenic $\mathrm{CD}^{+} \mathrm{T}$ lymphocytes to the central nervous system. The EBI2 signalling pathway (namely the receptor and $7 \alpha 25 \mathrm{HC}$ and its metabolic enzymes) has also been shown to respond to immune challenge in primary human monocyte-derived macrophages ${ }^{12}$. In the current study, we investigated EBI2 signalling and function in mouse astrocytes and aimed to demonstrate the role of EBI2 in cellular communication between astrocytes and macrophages.

Here, we found that in response to LPS, primary mouse astrocytes significantly down-regulated mRNA expression of EBI2. Our data also indicated that mouse astrocytes regulated mRNA levels of the $7 \alpha 25 \mathrm{HC}$ synthesising and degrading enzymes in response to a challenge with LPS. We can only speculate reasons for the relative 
slower temporal activations of CYP7B1 and HSD3B7 compared to $\mathrm{CH} 25 \mathrm{H}$ activation, which at present remains unclear. Importantly, this data is consistent with the mRNA expression of these enzymes in macrophages treated with LPS showing that $\mathrm{CH} 25 \mathrm{H}$ expression peaks after two hours, CYP7B1 mRNA can be observed over time of LPS treatment and CYP27A1 and HSD3B7 transcripts are down regulated within two or six hours ${ }^{12}$.

We found that upregulation of the synthesising enzymes was accompanied by an upregulation of $7 \alpha 25 \mathrm{HC}$ levels in mouse astrocyte conditioned media following LPS treatment. The findings that LPS increased the levels of $7 \alpha 25 \mathrm{HC}$ in mouse astrocyte conditioned media was associated with the ability of this media to promote migration of macrophages. We also found that conditioned media obtained from LPS-stimulated astrocytes induced macrophage migration that was partially attenuated with the EBI2 antagonist NIBR189. Thus, regulation of the EBI2 signalling pathway (both the receptor and enzymes that control levels of the ligand) in astrocytes appears to promote the migration of macrophage cells.

Taken together, the data indicates that molecules in the EBI2 signalling pathway are sensitive to pro-inflammatory signals, and that EBI2 plays a role in communication between astrocytes and macrophages.

\section{Methods}

Cell Culture. All animal procedures were approved by the institutional ethics committee (Novartis Pharma, Basel Switzerland and Trinity College Dublin, Ireland). Methods were carried out in accordance with the approved guidelines. Mouse astrocyte ${ }^{27-30}$ and mouse macrophage cells (RAW264.7) ${ }^{12}$ were cultured as we have described before. Prior to stimulation, astrocytes were starved in serum-free media for 4 hours. Astrocytes were then treated with LPS $\left(100 \mathrm{ng} / \mathrm{ml}\right.$, Sigma) with or without the EBI2 antagonist (NIBR189, Novartis) ${ }^{31}$ and/or $7 \alpha 25 \mathrm{HC}$ (prepared as $10 \mathrm{mM}$ stock in $90 \% \mathrm{DMSO}$ ).

Real Time Quantitative Polymerase Chain Reaction (RT-qPCR). For mRNA expression analysis, after treatment, astrocytes were washed twice with PBS, and either directly scraped or the cell pellet was resuspended in RA1 lysis buffer (Machery-Nagel, 740961) supplemented with $10 \% \beta$-mercapthoethanol and frozen at $-80^{\circ} \mathrm{C}$. RNA was isolated using the RNeasy Mini Kit (Qiagen Hilden, Germany). After reverse transcription of mRNA $\left(10 \mathrm{~min}, 25^{\circ} \mathrm{C} ; 120 \mathrm{~min}, 37^{\circ} \mathrm{C} ; 5 \mathrm{sec}, 85^{\circ} \mathrm{C}\right)$ using the High Capacity cDNA Reverse Transcription Kit (Applied Biosystems, Darmstadt, Germany), qRT-PCR was performed with the 7900HT Fast Real-Time PCR System (Applied Biosystems) according to the Standard Thermal Cycler Protocol $\left(2 \mathrm{~min}, 50^{\circ} \mathrm{C} ; 10 \mathrm{~min}, 95^{\circ} \mathrm{C}\right.$; 40 cycles $15 \mathrm{sec}, 95^{\circ} \mathrm{C}$ and $1 \mathrm{~min}, 60^{\circ} \mathrm{C}$ ). TaqMan Gene Expression Assays used the following FAM dye-labeled TaqMan mouse probes: EBI2 (Mm02620906-s1), CH25H (Mm00515486-s1), CYP7B1 (Mm00505894-g1), HSD3B7 (Mm01545399), HPRT1 (Mm01545399-m1) (Applied Biosystems). Applied Biosystems provide the context sequence (a 25 nucleotide sequence within which the probe sequence will lie) and within the details section of each of the assays provide a RefSeq, assay location nucleotide and amplicon length. For each of these assays the above mentioned context sequence can be found by counting 12 nucleotides on either side of the assay location nucleotide on the RefSeq (within this stretch of 25 nucleotides). The amplicon context sequence can similarly be found by counting the number of nucleotides corresponding to the amplicon length on either side of the assay location nucleotide on the RefSeq. Within this stretch of nucleotides on the Refseq the amplicon is located. Each condition was run in quadruplicates, with each quadruplicate RNA sample run in duplicates and the experiments were repeated 3 times. The threshold was set manually for all samples. The analysis was performed with the SDS 2.3 software. The relative expression of EBI2, CH25H, CYP7B1 and HSD3B7 to the reference gene (HPRT1) was determined.

Ultra-high performance liquid chromatography and tandem mass spectrometry (UHPLC-MS/MS). For mass spectroscopy the oxysterol standards and internal standards were purchased from Avanti Polar Lipis: $25 \mathrm{HC}, 27 \mathrm{HC}, 24 \mathrm{SHC}, 25 \mathrm{HC}-\mathrm{d} 6,27 \mathrm{HC}-\mathrm{d} 6,24(\mathrm{R} / \mathrm{S}) \mathrm{HC}-\mathrm{d} 7,7 \alpha 25 \mathrm{HC}, 7 \alpha 27 \mathrm{HC}, 7 \alpha 24 \mathrm{SHC}, 7 \beta 25 \mathrm{HC}, 7 \beta 27 \mathrm{HC}$, $7 \propto 25 \mathrm{HC}-\mathrm{d} 6,7 \propto 27 \mathrm{HC}-\mathrm{d} 6,7 \propto 24(\mathrm{R} / \mathrm{S}) \mathrm{HC}-\mathrm{d} 7$. Butylated hydroxytoluene (BHT) was purchased from SAFC (Sigma). Thirty million cells in $1 \mathrm{ml}$ of $\mathrm{H}_{2} \mathrm{O}$ containing a mixture of deuterated internal standard compounds and $200 \mu \mathrm{M}$ BHT were lysed using a Precellys ${ }^{\circledR} 24$ (Bertin Technologies, France) before extraction. To extract the oxysterols the lysed cells or $1 \mathrm{ml}$ culture media were gradually mixed with $9 \mathrm{ml}$ of EtOH by shaking and $4{ }^{\circ} \mathrm{C}$ cooling to allow slow precipitation. The extracts were then dried under $\mathrm{N}_{2}$ steam at $40^{\circ} \mathrm{C}$. The residue was resuspended in $100 \mu \mathrm{lEtOH} / \mathrm{H}_{2} \mathrm{O}$ and $10 \mu \mathrm{l}$ were injected onto a reverse-phase column for the ultra-high performance liquid chromatography and tandem mass spectrometry (UHPLC-MS/MS) analyses of oxysterols. The analyses were carried out on a Nexera UHPLC system (Shimadzu, Japan) coupled to a QTrap ${ }^{\circledR} 6500$ (ABSciex, Framingham, USA) mass spectrometer. Chromatographic separation was achieved using an Acquity UPLC ${ }^{\circledR}$ BEH C18 column $(100 \times 2.1 \mathrm{~mm}$, particle size $1.7 \mu \mathrm{m})$ with a VanGuard ${ }^{\mathrm{TM}}$ pre-colum Acquity UPLC ${ }^{\circledR}$ BEH C18 $(5 \times 2.1 \mathrm{~mm}$, particle size $1.7 \mu \mathrm{m})$ (Waters Assoc., USA). For the separation of monohydroxylated cholesterols, the mobile phases were delivered at a flow rate of $600 \mu \mathrm{l}$ and a column temperature of $55^{\circ} \mathrm{C}$. Mobile phase A consisted of $5 \% \mathrm{MeOH}$ in $\mathrm{H}_{2} \mathrm{O}$ and $0.1 \%$ formic acid (FA), whereas phase $\mathrm{B}$ of $\mathrm{MeOH} /$ acetonitrile (ACN) and $0.1 \%$ FA. The gradient program was composed of three isocratic steps: $6 \mathrm{~min}, 65 \%$ in phase $\mathrm{B}$ followed by $7 \mathrm{~min}, 70 \%$ in phase B and $2 \mathrm{~min}, 97 \%$ in phase B. For the separation of the dihydroxylated cholesterols, a 10 min linear gradient program from $73-97 \%$ in phase B was applied with a flow rate of $400 \mu \mathrm{l} / \mathrm{min}$ and a column temperature of $55^{\circ} \mathrm{C}$. Mobile phase A consisted of $5 \% \mathrm{MeOH}$ in $\mathrm{H}_{2} \mathrm{O}$ and $0.1 \%$ FA whereas phase $\mathrm{B}$ of $\mathrm{MeOH}$ and $0.1 \%$ FA. The mass spectrometer was equipped with an electrospray ionization source. The instrument was operated in a positive ion mode. Following MS/MS transitions were applied for the detection of oxysterols: $\mathrm{m} / \mathrm{z} 385 \rightarrow \mathrm{m} / \mathrm{z} 157$ and 159 for the mono-hydroxylated cholesterols and $\mathrm{m} / \mathrm{z} 383 \rightarrow \mathrm{m} / \mathrm{z} 157$ and 159 for dihydroxylated cholesterols.

Migration Assays. Migration of mouse macrophages (RAW264.7) was conducted at $37^{\circ} \mathrm{C}$ and $5 \% \mathrm{CO}_{2}$ in a humidified incubator using the xCELLigence ${ }^{\circledR}$ directed migration platform (Roche Applied Biosciences) with 
16-well CIM-plates as we have described previously ${ }^{22}$. In brief, the migration of cells through the electrode array from the upper to lower chambers is measured by an increase in impedance. The bottom chamber of the plate was filled with either astrocyte conditioned media or serum free media supplemented with $7 \alpha 25 \mathrm{HC}(0.01 \mu \mathrm{M})$ with or without EBI2 antagonist NIBR189 $(10 \mu \mathrm{M})$. Cell migration was recorded immediately after the plate was re-inserted and recorded for 2 hours.

\section{References}

1. Hannedouche, S. et al. Oxysterols direct immune cell migration via EBI2. Nature 475, 524-527 (2011).

2. Birkenbach, M., Josefsen, K., Yalamanchili, R., Lenoir, G. \& Kieff, E. Epstein-Barr virus-induced genes: first lymphocyte-specific G protein-coupled peptide receptors. J Virol 67, 2209-2220 (1993).

3. Liu, C. et al. Oxysterols direct B-cell migration through EBI2. Nature 475, 519-523 (2011).

4. Russell, D. W. Oxysterol biosynthetic enzymes. Biochim Biophys Acta 1529, 126-135 (2000).

5. Gatto, D., Wood, K. \& Brink, R. EBI2 operates independently of but in cooperation with CXCR5 and CCR7 to direct B cell migration and organization in follicles and the germinal center. J Immunol 187, 4621-4628 (2011).

6. Pereira, J. P., Kelly, L. M., Xu, Y. \& Cyster, J. G. EBI2 mediates B cell segregation between the outer and centre follicle. Nature 460, $1122-1126$ (2009).

7. Rosenkilde, M. M. et al. Molecular pharmacological phenotyping of EBI2. An orphan seven-transmembrane receptor with constitutive activity. J Biol Chem 281, 13199-13208 (2006).

8. Benned-Jensen, T. et al. Ligand Modulation of the Epstein-Barr Virus-induced Seven-transmembrane Receptor EBI2: Identification of a Potent and Efficacious Inverse Agonist. J Biol Chem 286, 29292-29302 (2011).

9. Benned-Jensen, T. et al. Small molecule antagonism of oxysterol-induced Epstein-Barr virus induced gene 2 (EBI2) activation. FEBS Open Bio 3, 156-160 (2013).

10. Yi, T. et al. Oxysterol Gradient Generation by Lymphoid Stromal Cells Guides Activated B Cell Movement during Humoral Responses. Immunity 37, 535-548 (2012).

11. Diczfalusy, U. et al. Marked upregulation of cholesterol 25-hydroxylase expression by lipopolysaccharide. J Lipid Res 50, 2258-2264 (2009).

12. Preuss, I. et al. Transcriptional regulation and functional characterization of the oxysterol/EBI2 system in primary human macrophages. Biochem Biophys Res Commun 2014, 663-668 (2014).

13. Bauman, D. R. et al. 25-Hydroxycholesterol secreted by macrophages in response to Toll-like receptor activation suppresses immunoglobulin A production. Proc Natl Acad Sci USA 106, 16764-16769 (2009).

14. Liu, S. Y. et al. Interferon-inducible cholesterol-25-hydroxylase broadly inhibits viral entry by production of 25 -hydroxycholesterol. Immunity 38, 92-105 (2013).

15. Blanc, M. et al. The transcription factor STAT-1 couples macrophage synthesis of 25-hydroxycholesterol to the interferon antiviral response. Immunity 38, 106-118 (2013).

16. Daugvilaite, V., Arfelt, K. N., Benned-Jensen, T., Sailer, A. W. \& Rosenkilde, M. M. EBI2-targeting oxysterols: a moving story. Eur J Immunol 2014, 201444493 (2014).

17. Norregaard, K., Benned-Jensen, T. \& Rosenkilde, M. M. EBI2, GPR18 and GPR17-three structurally related, but biologically distinct 7TM receptors. Curr Top Med Chem 11, 618-628 (2011)

18. Heinig, M. et al. A trans-acting locus regulates an anti-viral expression network and type 1 diabetes risk. Nature 467, 460-464 (2010).

19. Leoni, V., Lutjohann, D. \& Masterman, T. Levels of 7-oxocholesterol in cerebrospinal fluid are more than one thousand times lower than reported in multiple sclerosis. J Lipid Res 46, 191-195 (2005).

20. Ye, S. et al. Protein interaction for an interferon-inducible systemic lupus associated gene, IFIT1. Rheumatology (Oxford) 42, $1155-1163$ (2003).

21. Reboldi, A. et al. 25-Hydroxycholesterol suppresses interleukin-1-driven inflammation downstream of type I interferon. Science 345, 679-684 (2014).

22. Rutkowska, A., Preuss, I., Gessier, F., Sailer, A. W. \& Dev, K. K. EBI2 regulates intracellular signaling and migration in human astrocytes. Glia 63, 341-351 (2015).

23. Schule, R. et al. Marked accumulation of 27-hydroxycholesterol in SPG5 patients with hereditary spastic paresis. J Lipid Res 51, 819-823 (2010)

24. Sofroniew, M. V. Astrocyte barriers to neurotoxic inflammation. Nat Rev Neurosci 16, 249-263 (2015).

25. Schroder, K. et al. Conservation and divergence in Toll-like receptor 4-regulated gene expression in primary human versus mouse macrophages. Proc Natl Acad Sci USA 109, E944-953 (2012).

26. Chalmin, F., et al. Oxysterols regulate encephalitogenic CD4(+) T cell trafficking during central nervous system autoimmunity. $J$ Autoimmun 56, 45-55 (2015).

27. Osinde, M., Mullershausen, F. \& Dev, K. K. Phosphorylated FTY720 stimulates ERK phosphorylation in astrocytes via S1P receptors. Neuropharmacology 52, 1210-1218 (2007).

28. Mullershausen, F. et al. Phosphorylated FTY720 promotes astrocyte migration through sphingosine-1-phosphate receptors. $J$ Neurochem 102, 1151-1161 (2007).

29. Healy, L. M. et al. Pathway specific modulation of S1P1 receptor signalling in rat and human astrocytes. Br J Pharmacol 169, 1114-1129 (2013).

30. Elain, G., Jeanneau, K., Rutkowska, A., Mir, A. K. \& Dev, K. K. The selective anti-IL17A monoclonal antibody secukinumab (AIN457) attenuates IL17A-induced levels of IL6 in human astrocytes. Glia 62, 725-735 (2014).

31. Gessier, F. et al. Identification and Characterization of Small Molecule Modulators of the Epstein-Barr Virus-Induced Gene 2 (EBI2) Receptor. J Med Chem 57, 3358-3368 (2014).

\section{Acknowledgements}

The authors are grateful to Inga Preuss for experimental support. This work was supported by Irish Research Council (IRC) - Enterprise Partnership Scheme (EPS) with Novartis Pharma, Basel, Switzerland.

\section{Author Contributions}

A.R. performed experiments and analysed the data. I.C., J.Z. and S.A.O. performed UHPLC-MS/MS experiments and analysed the data. A.W.S and K.K.D. designed the study. A.R., A.W.S. and K.K.D. wrote the paper and prepared the figures. A.R. is an Irish Research Council (IRC) - Enterprise Partnership Scheme (EPS) PhD Fellow and S.A.O is a Trinity College Dublin PhD Fellow. 


\section{Additional Information}

Competing financial interests: Andreas W. Sailer, Isabelle Christen and Juan Zhang are employees of Novartis Pharma, Basel, Switzerland.

How to cite this article: Rutkowska, A. et al. The EBI2 signalling pathway plays a role in cellular crosstalk between astrocytes and macrophages. Sci. Rep. 6, 25520; doi: 10.1038/srep25520 (2016).

(c) (i) This work is licensed under a Creative Commons Attribution 4.0 International License. The images or other third party material in this article are included in the article's Creative Commons license, unless indicated otherwise in the credit line; if the material is not included under the Creative Commons license, users will need to obtain permission from the license holder to reproduce the material. To view a copy of this license, visit http://creativecommons.org/licenses/by/4.0/ 